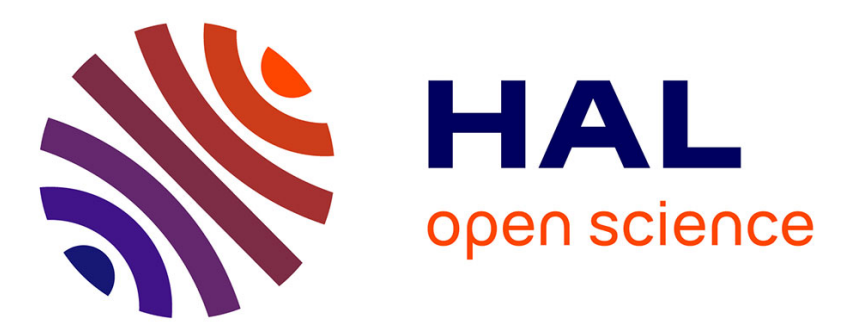

\title{
Magnetism of the rare earth, 3d - Theoretical review
}

\author{
M. Cyrot, D. Gignoux, F. Givord, M. Lavagna
}

\section{To cite this version:}

M. Cyrot, D. Gignoux, F. Givord, M. Lavagna. Magnetism of the rare earth, 3d - Theoretical review. Le Journal de Physique Colloques, 1979, 40 (C5), pp.C5-171-C5-176. 10.1051/jphyscol:1979563 . hal-01896507

\section{HAL Id: hal-01896507 https://hal.science/hal-01896507}

Submitted on 16 Oct 2018

HAL is a multi-disciplinary open access archive for the deposit and dissemination of scientific research documents, whether they are published or not. The documents may come from teaching and research institutions in France or abroad, or from public or private research centers.
L'archive ouverte pluridisciplinaire HAL, est destinée au dépôt et à la diffusion de documents scientifiques de niveau recherche, publiés ou non, émanant des établissements d'enseignement et de recherche français ou étrangers, des laboratoires publics ou privés. 


\title{
Magnetism of the rare earth, 3d - Theoretical review
}

\author{
M. Cyrot, D. Gignoux, F. Givord and M. Lavagna \\ Laboratoire Louis-Néel, CNRS, 166X, 38042 Grenoble Cedex, France
}

\begin{abstract}
Résumé. - Les composés terre rare-métal de transition présentent une large gamme de propriétés tout à fait originales. Afin d'expliquer leurs comportements magnétiques, il est nécessaire de connaître leur structure électronique. Nous rappelons les calculs existants et donnons la forme générale de la densité d'états de ces composés

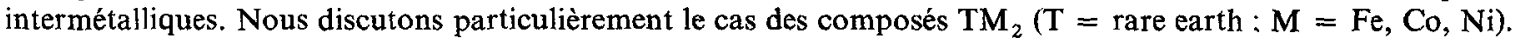
$\mathrm{La}$ série $\mathrm{TCO}_{2}$ présente le comportement le plus original puisque le cobalt peut ou non porter un moment magnétique suivant la terre rare. Nous rappelons les différentes théories élaborées au sujet des $\mathrm{TCo}_{2}$, et expliquons leur comportement magnétique par la position du niveau de Fermi dans une large décroissance de la densité d'états. Il s'agit d'un métamagnétisme d'électrons itinérants. Certains résultats expérimentaux que nous rappelons le confirment. Le comportement dit anormal d'autres composés avec le cobalt peut être expliqué de façon analogue.
\end{abstract}

\begin{abstract}
Compounds of rare earth and transition metals exhibit unusual and quite different behaviour. In order to explain the large variety of magnetic behaviour, we show that one needs the electronic structure in these compounds. We report on the existing calculations and give the general shape of the density of states in these intermetallic compounds. We discuss particularly the case of the $\mathrm{TM}_{2}$ compounds $(\mathrm{T}=$ rare earth $; \mathrm{M}=\mathrm{Fe}$, $\mathrm{Co}, \mathrm{Ni}$ ). The most unusual behaviour appears with the cobalt compounds where the cobalt within the rare earth series can be magnetic or not. We review the theories on the $\mathrm{TCo}_{2}$ series and explain the magnetic behaviour by the position of the Fermi level in this compound in a steep decrease of the density of states. This leads to a kind of collective metamagnetism. This is confirmed by experiments that we review. Unusual behaviour of other compounds with cobalt can be understood following the same lines as $\mathrm{TCo}_{2}$ and is discussed.
\end{abstract}

1. Introduction. - Intermetallic compounds between rare earth and $3 \mathrm{~d}$ elements have received much attention in the last years and many review articles appear [1]. Reasons are numerous. First rare earths possess large magnetic moment, while transition metals have high Curie temperature. These two properties are interesting in the same material and one finds good permanent magnet in this class of compounds. Secondly the chemical similarities of rare earth elements allow to substitute themselves one another in a given material. The main consequence is then to change the internal field seen by the d conduction electrons. Thus it permits to study the d magnetism in a clear way. This fundamental interest of intermetallic compounds is increased by the fact that first their total number is enormous and secondly it has advantage over solid solutions where disorder often unclears the results.

Barbara et al. [1] devide the compounds in two main groups. First, compounds whose magnetic properties are determined primarly by transition metals and secondly those determined mainly by rare earth metals. The first group are those rich in transition metal whose structure is related to the $\mathrm{CaCu}_{5}$ type. In general the transition metal bears a magnetic moments except $\mathrm{TCO}_{2}, \mathrm{TNi}_{5}, \mathrm{~T}_{2} \mathrm{Ni}_{7}, \mathrm{TNi}_{3}, \mathrm{TNi}_{2}$. When the transition metal is magnetic, the coupling between rare-earth and transition metal spins is always antiparallel. It depends neither on atomic environment, nor on interatomic distances, nor on conduction electron concentration. This makes parallel coupling between magnetic moment for the first part of the rare earth series and antiparallel for the second part. As we mention, $\mathrm{Ni}$ is non magnetic in many cases and $\mathrm{TCO}_{2}$ has particular properties that we will discuss in detail in the following. The second group contains the rare earth rich compounds. The cristallographic structure can be described from a trigonal prism. There is no magnetic moments on $\mathrm{Ni}$ and $\mathrm{Co}$, and compounds with iron do not exist. Critical temperature are much smaller, and magnetic properties bear resemblances with rare earth metals. Thus we will focus our attention in this review on the first group. Within this group, we can discuss three cases. The transition metal has a well defined magnetic moment, or it has no moment at all, or we can have the bordeline case where the moments appear under some conditions. This is the case of the series $\mathrm{TCO}_{2}$ and the case of pseudo-binary systems where we mixed two rare earth metals as $\mathrm{Y}_{x} \mathrm{Gd}_{1-x} \mathrm{Co}_{2}$. As we are interested by the appearance of $3 \mathrm{~d}$ magnetism, we will mainly be concerned by these bordeline cases.

2. The model of Bloch and Lemaire. - The first attempt towards an understanding of the magnetic properties of intermetallic compounds is due to Bloch 
and Lemaire [2]. Their purpose was the explanation of the paramagnetic susceptibility of $\mathrm{RCo}_{2}$ compounds and they discussed the following model. The compound is made of two types of atoms $R$ and $M$. R, the rare earth atom is assumed to possess a welllocalized magnetic moment and $M$ the transition metal atom gives rise to $d$ energy bands. The coupling between moment and electron leads to an exchange enhanced paramagnetic susceptibility. They introduced it by means of molecular field coefficients which are related to exchange interaction coefficients $J_{\mathrm{RR}}$ and $J_{\mathrm{RM}}$.

Values of $J_{\mathbf{R R}}$ and $J_{\mathbf{R M}}$ obtained from susceptibility measurements, are given in table I. The value of the coupling between rare earth and transition metal is nearly three times the value between rare earth.

Table I. - Exchange interaction coefficients of $\mathrm{TCO}_{2}$ compounds.

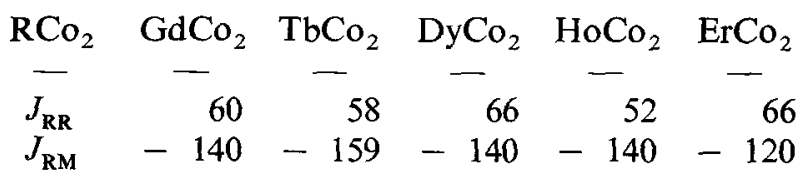

Bloch et al. [3] extended the previous model to study the order of the transition in the $\mathrm{RCo}_{2}$ series. They assumed that the structure of the $\mathrm{d}$ band and the position of the paramagnetic Fermi level is the same within the series. The free energy due to $d$ electrons can always be expanded in powers of the magnetization which would be for instance that of $\mathrm{YCo}_{2}$ :

$$
F_{\mathrm{d}}=F_{\mathrm{d}}^{0}+A(T) M_{\mathrm{d}}^{2}+\frac{1}{4} B(T) M_{\mathrm{d}}^{4} .
$$

In a Landau theory, the sign of $B(T)$ is related to the order of the transition and $A(T)=\chi_{\mathrm{d}^{-1}}(T)$, is the inverse of the susceptibility of the $d$ band which is known experimentally.

The magnetization $M_{\mathrm{d}}$ of $\mathrm{YCo}_{2}$ in an applied field $H$ is given by :

$$
M_{\mathrm{d}}(T)=\chi_{\mathrm{d}}(T) H-B(T)\left[\chi_{\mathrm{d}}(T)\right]^{4} H^{3} .
$$

Thus, measurement of the $H^{3}$ term in $M_{\mathrm{d}}(T)$ gives direct information about $B(T)$. High field measurement gives a negative value of $B(T)$ up to $300 \mathrm{~K}$ where, within experimental error, $B(T)$ is zero.

The negative value of $B(T)$ in $\mathrm{YCo}_{2}$ has the following consequence. If $\mathrm{Y}$ is replaced by a magnetic rare earth, within mean field theory, we add an internal field and the Gibbs potential becomes :

$$
\begin{aligned}
& G=F_{\mathrm{d}}-H M_{\mathrm{d}}-N k T \log \frac{\operatorname{sh}\left(J+\frac{1}{2}\right) y}{\operatorname{sh} y / 2} \\
& y=\frac{g \mu_{\mathrm{B}} H+n_{\mathrm{RM}} M_{\mathrm{d}}}{k T}
\end{aligned}
$$

expansion of the last term gives negligible correction to $B(T)$ and the transition is first order when occurring at low temperature and second order when occurring at higher temperature. Experimentally, $B(T)$ must change sign near $200 \mathrm{~K}$ as Dy Ho and Er compounds exhibit a first order transition while $\mathrm{Tb}$ and $\mathrm{Gd}$ a second order one.

These authors make an attempt to justify this phenomenological approach by a microscopic theory. They applied Stoner theory to the Cobalt $\mathrm{d}$ band. The coefficient of the Landau expansion are given within this theory as a function of various derivatives of the density of states at the Fermi Level and they suggest that various properties would be well explained : change of sign of $B(T)$, maximum in the susceptibility of $\mathrm{YCo}_{2} \ldots$ if one takes the Fermi level to be in a minimum of density of states.

3. Density of states in $R M_{2}$ compounds $(M=F e$, Co, Ni). - In order to answer the question of Bloch et al. about the position of the Fermi level within the band in $\mathrm{YCO}_{2}$, and more generally to understand the magnetic properties of the transition atom in these compounds, it is important to know the density of states. For pure rare earth, the density of states is much like that of transition metals where numerous calculations exist [5]. Cyrot and Lavagna [4] used the same kind of method assuming that the rare earth atom behaves as a transition atom with $5 \mathrm{~d}$ electrons. Thus the calculation is the same as an ordered alloy of transition metals. Within this scheme the density of states varies little in a series $R_{2}$ when one varies the rare earth metal. This stems from two facts : first the atomic energy of the $5 \mathrm{~d}$ state changes little within the rare earth series, secondly the transfer integral from the rare atom to the transition atom is the same within the series because the configuration and thus the Slater $q$ value, remains constant. The rare earth atomic level (or $\mathrm{Y}$ one) is always upper than the $\mathrm{Ni}$, Co or $\mathrm{Fe}$ one and the transfer of electron is from the rare earth to the transition metal. The local density of states on the

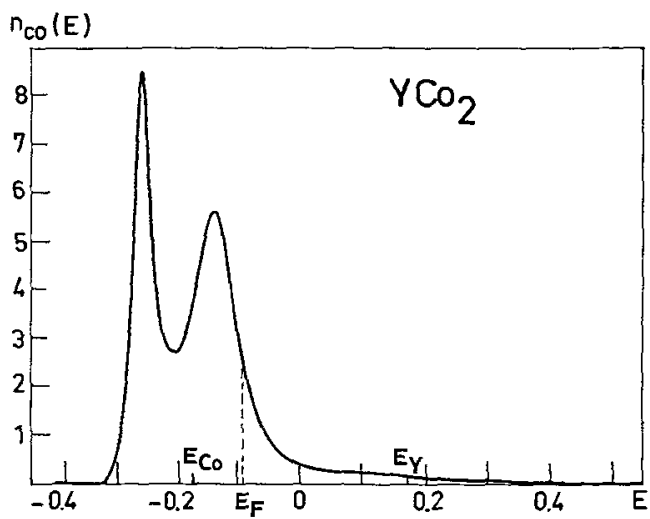

Fig. 1. - Local density of states at the $\mathrm{Co}^{\text {in }} \mathrm{YCo}_{2}\left(n_{\mathrm{Co}}(E)\right.$ in states $\mathrm{orb}^{-1} \operatorname{spin}^{-1} \mathrm{Ryd}^{-1} ; E$ in Ryd). 
transition atom is much like that in pure metal (figure 1). On the contrary the local density of states on the rare earth atom is much lower and wider than in pure metal. This is due to much smaller interatomic distance in compounds than in rare earth metals.

For Ni compounds, the charge transfer is large enough such that the $d$ band is full and the Fermi level lies in the part of the density mainly due to the rare earth atom. The density of states at the Fermi level is small and $\mathrm{Ni}$ is never magnetic. For Cobalt compounds, this transfer towards Cobalt makes the $d$ band nearly full and the Fermi level lies in the steep decrease between that part of the density mainly due to the transition atom and that much lower mainly due to the rare earth. Experimental studies of the resistivity performed on $(\mathrm{Ho}, \mathrm{Y}) \mathrm{Co}_{2}$ confirm this assumption [6]. Slight change of the filling or of others parameters leads to large modification of the density of states at the Fermi level. The Stoner criterium is nearly fulfilled for $\mathrm{YCO}_{2}$. For iron compounds, the transfer lets the Fermi level within this part of the density mainly due to transition atom and Iron remains magnetic in all compounds.

4. Electronic properties of $\mathrm{YCo}_{2} .-\mathrm{YCO}_{2}$ is a Pauli paramagnet. The susceptibility increases at low temperature and have a maximum near $250 \mathrm{~K}$. The value of $\gamma$, the electronic contribution to the specific heat, is high. These properties have been explained by Bloch et al. using the Stoner model. They showed that one can obtain a good fit with reasonable value of the derivative of the density of states at the Fermi level. As calculation of the density of states shows that Stoner criterium is nearly satisfied for $\mathrm{YCO}_{2}$, we suggest that paramagnons effects should be important. First the calculated value of $\gamma$ [4] is in good accordance with the experimental one for $\mathrm{YFe}_{2}$ and $\mathrm{YNi}_{2}$ (respectively 6.5 and 6 for iron and 1.8 and 1.8 for nickel [in $\left.\mathrm{mJ} \mathrm{mole}^{-1} \mathrm{~K}^{-2}\right]$ ). The calculated value for $\mathrm{YCo}_{2}$ is much lower than the experimental one. Moreover, measurements of Muraoka [7] on pseudo binary alloys $\mathrm{Y}\left(\mathrm{Fe}_{1-x} \mathrm{Co}_{x}\right)_{2}$ show a maximum of $y$ for $x=0.9$ which is the concentration of appearance of magnetism. Secondly the susceptibility increases with temperature due to the positive value of the curvature of the density of states at the Fermi Level. Paramagnons effects make the $T^{2}$ coefficient of the expansion of $\gamma$ varie as the square of the Stoner factor. By comparison with $\mathrm{LuCo}_{2}$ which we expect much lower paramagnon effect, one would be able to test this point. Third, the best way to detect these effects is through the study of the resistivity at low temperature which has a large $T^{2}$ term. Such effect seems to have been observed recently by Ikeda [8]. Following this line, we would attribuate the maximum in the susceptibility of $\mathrm{YCO}_{2}$ to the fact that the spin fluctuation temperature is of order $200 \mathrm{~K}$ in accordance to the following discussion on itinerant metamagnetism.
5. Appearance of $3 \mathrm{~d}$ magnetism in the $\mathrm{RCO}_{2}$ series. When the rare earth is not magnetic, the Cobalt presents no moments and the compound is a Pauli paramagnet : $\mathrm{LuCo}_{2}$ [9], $\mathrm{YCo}_{2}$ [10]. The moment appears with the spin of the rare earth and is of order $1 \mu_{\mathrm{B}}$. The transition is second order except in $\mathrm{DyCo}_{2}$ [11], $\mathrm{HoCO}_{2}$ [10] and $\mathrm{ErCo}_{2}$ [12]. We described the phenomenological theory of Bloch et al. on this problem. We now discuss the theory of Cyrot and Lavagna which is based on the calculated density of states and which gives a Fermi level in a steep decrease of the density of states. The basic idea is Bloch and Lemaire's one : the moment on the Cobalt is induced by exchange. Introducing a phenomenological exchange constant $\Gamma$ between the spin of the rareearth $S_{z}$ and the spin of the delectron of the transition atom, in a simple Hartree-Fock treatment, the $\mathrm{d}$ band is split and the splitting between up and down spin is given by

$$
U m_{\mathrm{d}}+2 \mu_{\mathrm{B}} B_{\mathrm{a}}+\Gamma\left\langle S_{z}\right\rangle
$$

where $U$ is the interatomic coulomb interaction, $m_{\mathrm{d}}$ the d magnetization, $B_{\mathrm{a}}$ an applied field and $\left\langle S_{z}\right\rangle$ the average of the rare earth spin. The essence of the theory is that this effective field moves the Fermi energy of spin up in a low density of states region and the Fermi level of spin down in a high density of states. The generalized Stoner criterium for this case is :

$$
\frac{U}{\frac{1}{2 n \uparrow\left(E_{\mathrm{F}}\right)}+\frac{1}{2 n \downarrow\left(E_{\mathrm{F}}\right)}}>1
$$

which gives the instability towards a magnetic state for Cobalt atom. $n \downarrow$ increasing much more than $n \uparrow$ is decreasing, makes this inequality fulfilled in some cases and Co atom magnetizes in a discontinuous way (figure 2) ; this must be the case with Dy, Ho and Er. Such a Cobalt behaviour has been observed experimentally on $\mathrm{HoCO}_{2}$ by means of polarized neutron study [13, 14] (figure 3).

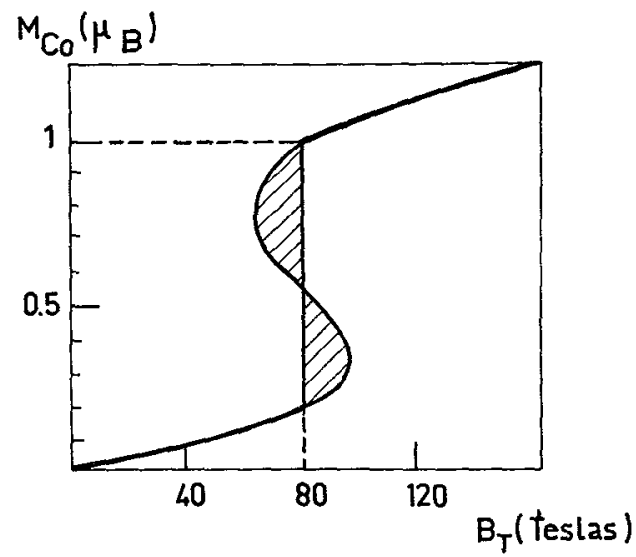

Fig. 2. - Theorical variation of the Cobalt magnetization as a function of $B_{\mathrm{T}}$ in $\mathrm{TCo}_{2}$. 


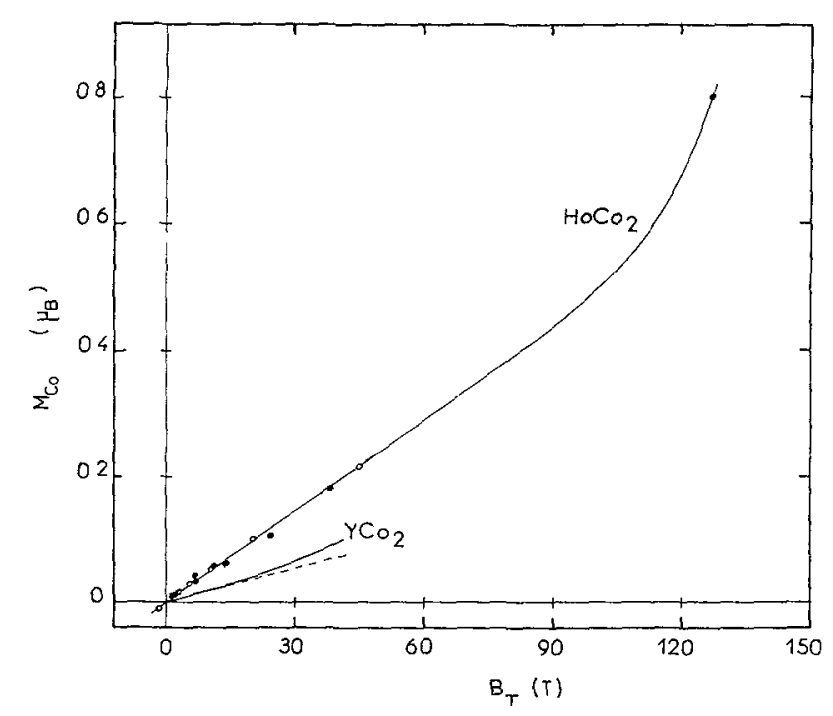

Fig. 3. - Experimental variation of the Cobalt magnetization as a function of $B_{\mathrm{T}}$ in $\mathrm{HoCO}_{2}$ and $\mathrm{YCO}_{2}$.

For $\mathrm{YCo}_{2}$, the authors have calculated the curve $m_{\mathrm{d}}$ as a function of the internal field to be discontinuous (figure 2). At low field, the magnetization is low and at a critical field $B_{\text {crit }}$ the magnetization jumps to a large value $m_{\text {crit }}$ as confirmed experimentally [14] (figure 3).

The internal field is given by the rare earth moments which are magnetized themselves by the $\mathrm{d}$ moment, the molecular field constant being

$$
\lambda=\frac{g-1}{2 g \mu_{\mathrm{B}}^{2}} \Gamma \text {. }
$$

Thus one has to solve selfconsistently the set of equations

$$
\begin{aligned}
& m_{\mathrm{d}}=f\left(\lambda m_{\mathrm{R}}, T\right) \\
& m_{\mathrm{R}}=g \mu_{\mathrm{B}} J \mathfrak{B}_{\mathrm{J}}\left(\frac{\lambda m_{\mathrm{d}}}{k T}\right)
\end{aligned}
$$

$m_{\mathrm{R}}$ is the magnetization of the rare earth. Graphical discussion (figure 6) will give the self consistent value for $m_{\mathrm{d}}$. In the high temperature region, there is no intersect and $m_{\mathrm{d}}$ equals zero. As one lowers the temperature, two behaviours can happen. First the two curves become tangent at the origin and the magnetization appears continuously. The transition is second order. In that case the Curie temperature $\theta_{\mathrm{c}}$ is given when the slope of equation (7a), which is the susceptibility of the $\mathrm{d}$ electrons at temperature $\theta_{\mathrm{c}}$, is equal to the slope of $(7 b)$, one obtains

$$
k \theta_{\mathrm{c}}=\frac{\Gamma^{2}(g-1)^{2} J(J+1)}{12 \mu_{\mathrm{B}}^{2}} \chi\left(\theta_{\mathrm{c}}\right)
$$

the second possibility is that the Brillouin curve $(7 b)$ intersects $(7 a)$ when one lowers temperature at the point $B_{\text {crit }}, M_{\text {crit }}$. In that case the magnetization

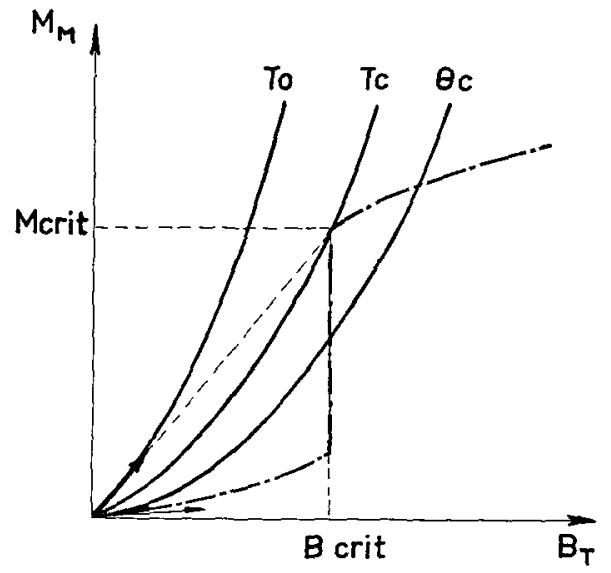

Fig. 4. - Definition of the first and second order temperatures in $\mathrm{TCO}_{2}$.

appears discontinuously and the transition is first order. A rough estimation of the Curie temperature is

$$
k T_{0}=\frac{\Gamma^{2}(g-1)^{2} J(J+1)}{12 \mu_{\mathrm{B}}^{2}} \frac{M_{\text {crit }}}{B_{\text {crit }}} .
$$

Discussion of the order of the transition will consist on the comparison between these two temperatures. If $\chi\left(\theta_{\mathrm{c}}\right)$ is smaller than $M_{\text {crit }} / B_{\text {crit }}$, the transition will be first order. As $\chi(\theta)$ increases with temperature in this domain, the transition will go from first order to second when going from $\mathrm{Er}$ to $\mathrm{Gd}\left(T_{\mathrm{c}}\right.$ going from $33 \mathrm{~K}$ to $395 \mathrm{~K}$ ). Calculated values along these lines are given in table II.

Table II. - Transition temperatures of the $\mathrm{RCo}_{2}$ compounds with heavy rare earths.

$\begin{array}{ccc}\mathrm{RCo}_{2} & T_{\mathrm{c}} \text { calculated in } \mathrm{K} & T_{\mathrm{c}} \text { exp. in } \mathrm{K} \\ - & - & - \\ \mathrm{DyCo}_{2} & 138 & 135 \\ \mathrm{HoCo}_{2} & 84 & 77 \\ \mathrm{ErCo}_{2} & 43 & 33\end{array}$

The first serie of rare earth presents the following problem : even for low $T_{\mathrm{c}}$, the transition remains second order. For this serie, one can argue that the susceptibility of the $\mathrm{d}$ band is higher than in the heavy rare earth because there is a slight change in the density of states due to a slight decrease of the $d$ atomic level of the rare earth. This decrease will increase the density of states at the Fermi level. This is experimentally shown by the slight variation of $\gamma$ in the series. Thus the susceptibility is also increased leading to a second order transition for light rare earth.

Cyrot et al. [4] also discussed the behaviour of these compounds under a magnetic field. The internal field necessary to induce the moment on Co in the Dy, Ho 
and Er compounds can be obtained by an external field. Thus applying magnetic field above $T_{c}$ can induce the transition for a critical value of the field.

6. $\mathbf{R F e}_{2}$ and $\mathbf{R N i}_{2}$ series. - In the series $\mathrm{RFe}_{2}$, calculation of the density of states gives a paramagnetic Fermi energy in a region of high density of states. Stoner criterium is fulfilled and Iron is always magnetic even if the rare earth metal is not. The value of the moment changes slightly along the series (figure 5) values of the Curie temperature can be calculated from equation (8) [4]. For a rough estimation, the susceptibility of the $d$ electrons can be approximated by a Curie-Weiss law which is given by the experimental result for $\mathrm{YFe}_{2}$. Table III tabulates the theoretical values of the Curie constant $C$ which give the experimental ordering temperatures of different $\mathrm{RFe}_{2}$ compounds. $C$ does not change much and is near to the experimental Curie constant of $\mathrm{YFe}_{2}\left(2 \mu_{\mathrm{B}} \mathrm{KT}^{-1}\right.$ at $\left.^{-2}\right)$. On the contrary for $\mathrm{RNi}_{2}$, calculation gives the Nickel to be non magnetic in all cases. Following the same line of approach, the Curie temperature is always given by equation (8). The susceptibility in that case is Pauli like and for an estimation that of $\mathrm{YNi}_{2}$. Table III gives the theorical values of the susceptibility $\chi$ which permit the experimental Curie temperatures of different $\mathrm{RNi}_{2}$

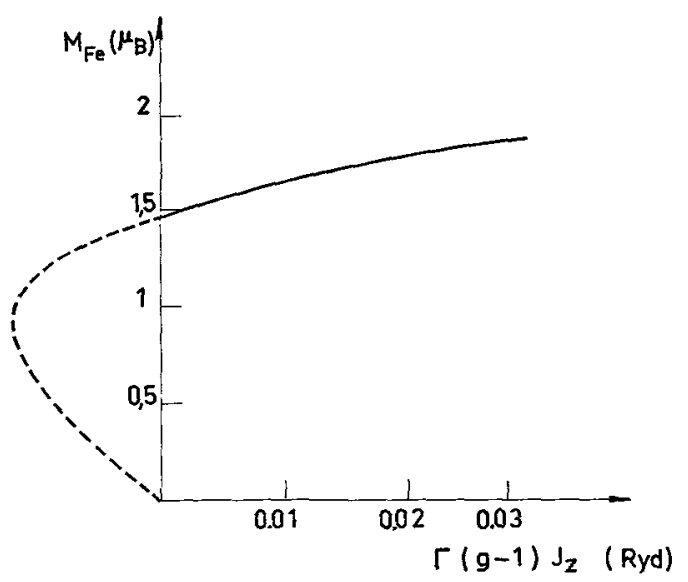

Fig. 5. - Theorical variation of the Iron magnetization as a function of $\Gamma(g-1) J_{z}$ in $\mathrm{TFe}_{2}$. compounds to be predicted. We note that this susceptibility does not change much and is near to the experimental value $\left(1.25 \mu_{\mathbf{B}} \mathrm{T}^{-1}\right.$ at $\left.^{-1}\right)$.

7. Other cases of itinerant metamagnetism. - The anomalous behaviour of Cobalt in the $\mathrm{TM}_{2}$ series has been ascribed to the particular position of the Fermi Level in a steep decrease. This situation can occur in other compounds. Let us quote $\mathrm{YCO}_{3}$ and $\mathrm{ThCO}_{5}$. In these cases, the Cobalt atoms can occupy two different cristallographical sites $\mathbf{M}^{\prime}$ and $\mathbf{M}^{\prime \prime}$. On site $\mathbf{M}^{\prime}$, Cobalt bears a magnetic moment and on site $\mathrm{M}^{\prime \prime}$, one has the particular position of the Fermi Level which gives properties of $\mathrm{YCo}_{2}$. The magnetization of these compounds has been analysed by Givord et al. [15] as the sum of that due to the $M^{\prime}$ site and that due to the $\mathbf{M}^{\prime \prime}$ site which presents a maximum at finite temperature due to the maximum of the susceptibility. Thus the total magnetization presents

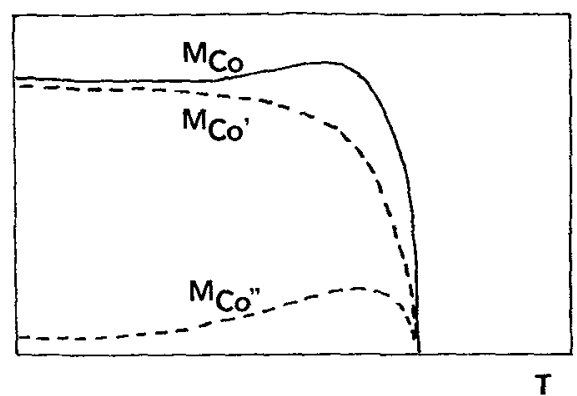

(a)

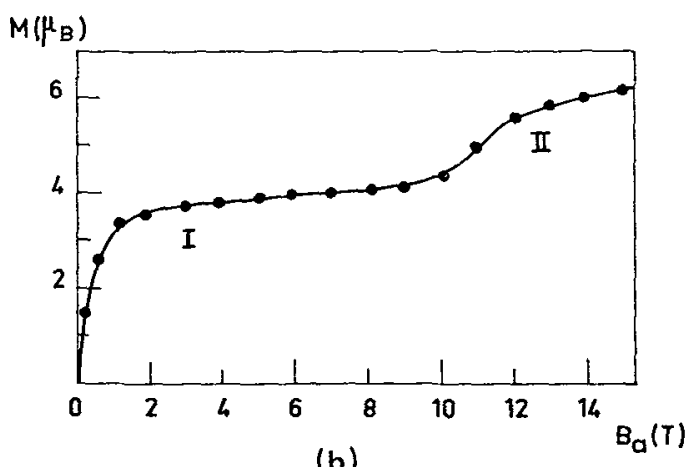

Fig. 6. - (a) Cobalt moment versus temperature in $\mathrm{ThCo}_{5}$; (b) Magnetization curve in $\mathrm{ThCo}_{5}$.

Table III. - Transition temperatures of $\mathrm{RFe}_{2}$ and $\mathrm{RNi}_{2}$.

\begin{tabular}{|c|c|c|c|c|}
\hline $\mathbf{R}$ & $\begin{array}{c}\mathbf{R F e}_{2} \\
\theta_{\mathbf{c}} \text { exp. in } \mathbf{K}\end{array}$ & $\begin{array}{c}\mathrm{RFe}_{2} \\
C \mu_{\mathrm{B}} \mathrm{KT}^{-1} \mathrm{at}^{-1}\end{array}$ & $\stackrel{\mathrm{RNi}_{2}}{\theta_{\mathbf{c}} \text { exp. in } \mathrm{K}}$ & $\chi 10^{-3} \mu_{\mathrm{BNi}} \mathrm{T}^{-1}$ at $^{-1}$ \\
\hline- & - & - & - & - \\
\hline $\mathrm{Sm}$ & 688 & 3.4 & 21 & 1.3 \\
\hline $\mathrm{Gd}$ & 782 & 1.9 & 85 & 1.5 \\
\hline $\mathrm{Tb}$ & 705 & 1.8 & 45 & 1.2 \\
\hline Dy & 638 & 1.5 & 30 & 1.2 \\
\hline Ho & 614 & 1.6 & 22 & 1.4 \\
\hline Er & 596 & 2.1 & 21 & 2.2 \\
\hline
\end{tabular}


a maximum at finite temperature (figure $6 a$ ). Magnetization curve (figure $6 b$ ) under magnetic field can be interpret along the same line. The magnetic moment on site $M^{\prime \prime}$ can be induced by an external magnetic field leading to a steep increase of the magnetization.

This anomalous behaviour is not limited to Cobalt compounds. In Nickel compounds with high concen- tration of Nickel, it is possible to lower the Fermi level down to the point where it enters in the part of the density of states mainly due to Nickel. The behaviour of figure $6 a$ has been obtained on $\mathrm{Y}_{2} \mathrm{Ni}_{17-\varepsilon}[16]$.

Acknowledgements. - We warmly thank R. Lemaire for stimulating discussions and various suggestions.

\section{References}

[1] See for instance the review articles :

Barbara, B., Gignoux, D., Givord, D., Givord, F. and Lemaire, R., Int. J. Magnetism 4 (1973) 77.

KIRChMAYR, H. R. and Poldy, C. A., J. Magn. Magn. Materials 8 (1978) 1.

Buschow, K. H. J., Reports Progr. Physics 40 (1977) 1179.

[2] Bloch, D. and Lemaire, R., Phys. Rev. B 2 (1970) 2648.

[3] Bloch, D., EDwards, D. M., Shimizu, M. and Voiron, J., J. Phys. F 5 (1975) 1217.

[4] Lavagna, M., Thesis Grenoble 1978.

Cyrot, M., Lavagna, M. (to be published).

[5] Gaspard, J. P., Cyrot-Lackmann, F., J. Phys. C, Solid State Phys. 6 (1973) 3077.

[6] Steiner, W., Gratz, E., Ortbauer, H. and Camen, H. W. (to be published).

[7] Muraoka, Y., Shiga, M. and Nakamura, Y., J. Phys. Soc. Japan 42 (1977) 2067.
[8] IKedA, K., J. Less-Common Metals 52 (1977) 101.

[9] Grvord, F. and Lemaire, R., Solid State Commun. 9 (1971) 341.

[10] Lemaire, R., Cobalt 33 (1966) 201.

[11] Givord, F. and ShaH, J. J., C.R. Hebd. Séan. Acad. Sci. Paris, B 274 (1972) 923.

[12] Petrach, G. and Mossbauer, R. L., Phys. Lett. 26A (1968) 403.

[13] Gignoux, D., Givord, F. and KobHLER, W. C., Physica 86-88B (1977) 165.

[14] Gignoux, D., Givord, F. and Schweizer, J., J. Phys. F 7 (1977) 1823.

[15] Givord, D., Laforest, J., Lemaire, R., Physica 86-88B (1977) 204.

[16] Grgnoux, D., Lemaire, R., Mol ho, P., Private communication. 